\title{
ADMINISTRACIÓN Y GLOBALIZACIÓN: UNA PERSPECTIVA PSICOLÓGICA
}

José Perea R. ${ }^{1}$

(Recibido el 02/10/2006, aceptado el 18/04/2007)

\begin{abstract}
RESUMEN
La temática general de la administración y la globalización tiene como propósito de abordarla como ejes basados en el conocimiento sistematizado o en la interpretación que en el siglo XX iniciaron y vienen marcando sea en una prospectiva emprendedora o apocalíptica la orientación del desarrollo de las organizaciones y de los seres humanos en el siglo XXI, es decir, en la dinámica de las organizaciones: comunidad, países o bloques continentales, y la dinámica personal. La importancia de las temáticas es haberse convertido en el centro de atención tanto en el nivel individual, organizacional, así como mundial, y que pretendemos tratarlas en una perspectiva psicológica flexible, presentándolas a manera de una propuesta de investigación exploratoria de enfoque post facto, en la cual la administración considerada como gestora del desarrollo de las organizaciones, dilucide si es la causa o efecto de la globalización; asimismo, el exponer los criterios que a manera de propuestas han sido expresados como alternativas propositivas para su afrontamiento en un criterio sistémico y en perspectiva global hacia la modernidad del siglo XXI de cambio organizacional y concertación.
\end{abstract}

Palabras claves: Administración, Globalización, Cambio organizacional, Concertación.

\begin{abstract}
The thematic general of the administration and the globalización must like intention to approach it like axes based on the systematized knowledge or the interpretation that in century xx initiated and comes marking is in an enterprising or apocalyptic prospectiva the direction of the development of the organizations and the human beings in century XXI, that is to say, in the dynamics of the organizations: community, continental countries or blocks, and personal dynamics. The importance of the thematic ones is to have turned in center of attention as much the individual level, organizacional like world-wide, and that we try to treat them in a flexible psychological perspective, presenting/displaying them to way of a proposal of exploratory investigation of approach post facto, in which the administration considered like manager of the development of the organizations, explain if it is the cause or effect of the globalización; also, exposing the criteria that to way of proposals have been expressed like propositive alternatives for their facing in a sistémico criterion and global perspective towards the modernity of century XXI of organizacional change and agreement.
\end{abstract}

Keywords: Administration, Globalización, Organizacional change, Agreement.

\footnotetext{
1 Docente de la Facultad de Psicología de la UNMSM. E-mail: jperear@unmsm.edu.pe
} 


\section{INTRODUCCIÓN}

El contenido del presente de trabajo tiene un marco referencial interdisciplinario, de las ciencias de la Administración y la Psicología, que a través de sus principales principios y/o técnicas permitan abordar las consecuencias de la globalización en las organizaciones de producción de bienes y/o servicios privadas y gubernamentales (industrias-empresasinstituciones) de países o bloques continentales, en la dinámica personal y laboral de los seres humanos con relación a su éxito, supervivencia o fracaso en la expectativa del nivel de calidad y futuro de vida.

Sea cual fuera la perspectiva o enfoque, la globalización produce consecuencias en las organizaciones y en las personas, que en una perspectiva psicológica se entenderá como situaciones/problemas que generan cambio, tomando como una de las opciones de logro la convivencia, con libertad y concertación que permita el desarrollo sostenible de la sociedad en su conjunto.

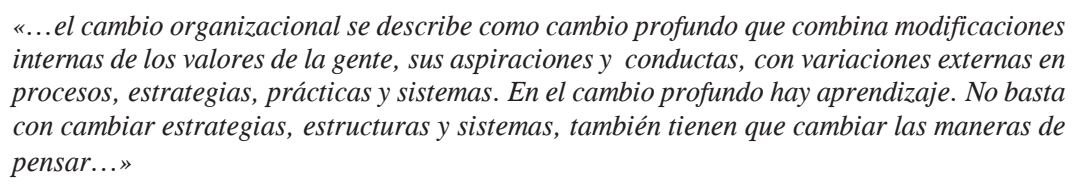
internas de los valores de la gente, sus aspiraciones y conductas, con variaciones externas en procesos, estrategias, prácticas y sistemas. En el cambio profundo hay aprendizaje. No basta con cambiar estrategias, estructuras y sistemas, también tienen que cambiar las maneras de pensar...»

Peter Senge, La danza del cambio (1) (2).

La temática exige de una explicitación focalizada, por un lado el de los contenidos de la Administración, y por otro lado, la aplicación de la Psicología en el ámbito organizacional al tratar las propuestas de atención y/o solución a las situaciones / problemas que viene generando la Globalización.

En esta perspectiva, el primer nivel de trabajo intenta delinear la naturaleza de la globalización, para luego tratar el contenido de los referentes más cercanos relacionados a los principios y técnicas de cada una de las funciones administrativas o procesos de gestión que desarrolla la Administración en las organizaciones, y en un tercer nivel de trabajo los tópicos como propuestas mediante el cambio con libertad y concertación.

\section{Planteamiento de la situación o problema}

En este primer nivel de trabajo para un entendimiento y un comprender objetivo, intentaremos dilucidar si una situación o un problema en la práctica de la Administración se convierte en relación causal o respuesta a la Globalización; a los efectos que influyen en la dinámica de las organizaciones o de las personas.

Es un saber del trabajo psicológico organizacional en el actual contexto globalizado, que toda organización está expuesta a situaciones o problemas que inciden en su desarrollo y que la no atención o solución oportuna, eficiente y eficaz por su administración o gestión, pueden convertirla o declararse en sujeto «afectado» por la globalización.

En este sentido, primero debemos clarificar y determinar que una situación representa aspectos nuevos, no previstos, provienen de afuera, no está y no es parte de la dinámica interna de la organización. 
Las situaciones son externas a la organización que pueden ser originadas por el contexto local donde se encuentra operando, o por una región connacional, continental o mundial.

Entre los aspectos que se consideran como una situación a la que está expuesta la organización, se encuentran los nuevos impuestos o arbitrios, nuevas alzas de intereses bancarios o aranceles, nuevas políticas gubernamentales de ampliación de cobertura de servicios o de producción de recursos naturales con horizonte exportador o de ampliación de beneficios laborales o sociales, políticas de comercialización con nuevas tendencias del mercado sectorial, nacional o internacional, aparición de nuevos productos, o de convenios o tratados comerciales internacionales resueltos o no ratificados.

Otros aspectos serían la discontinuidad de materiales o productos que la organización los considera como insumos, o que por efectos estacionales -ejemplo, fenómeno de El niñoproducen desabastecimiento inusual de recursos, y que los necesita en sus procesos de transformación de productos.

Lo particular o lo paradójico en estos tiempos de cambios acelerados, es que las situaciones que representan aspectos nuevos, no son percibidas o tomadas en cuenta, ni como referentes circunstanciales para un análisis preliminar, es decir no se les da la mínima importancia.

Estas situaciones al no ser atendidas por la administración o la gestión, se convierten en problemas, pasando a ser parte de la dinámica interna de la organización.

Ahora corresponde tratar sobre los problemas, que representan los aspectos que no responden a lo que está previsto, y que afectan a lo planificado o programado, que es lo conocido, que es parte de la dinámica interna de la organización.

Por tanto, los problemas no son acciones externas originadas por el contexto local donde se encuentra operando, o región connacional, continental o mundial, son aspectos que generalmente resultan de acciones de negligencia o impericia en la aplicación de los procedimientos para sus procesos de transformación que retardan o impiden la producción de bienes o servicios previstos.

Es pertinente anotar que los problemas de naturaleza funcional por recursos humanos, pueden ser producidos sea porque el personal no conoce el puesto de trabajo o no sabe sus funciones, o porque no quiere trabajar, que es más delicado o complejo.

Es necesario precisar, que los problemas en sus efectos limitantes o de frenaje para la producción, no sólo son de naturaleza funcional relacionada a los recursos humanos, también pueden corresponder a los recursos de naturaleza económica y financiera, a recursos organizacionales: estructura orgánica, reglamentos, manuales, etc., o a los recursos físicos: infraestructura, equipos y maquinarias, entre otros.

Los efectos de las situaciones producidas por aspectos externos y no atendidas, o por los problemas por aspectos generados en la dinámica interna y que no son solucionados, evidencian que la administración o la gestión, no las está afrontando en forma oportuna, eficiente y efectiva, no asume conscientemente su responsabilidad ante la organización, y ella a su vez, no asume su responsabilidad ante la comunidad local, regional o nacional.

En este primer planteamiento, los efectos de las situaciones o problemas no atendidas o solucionadas son consecuencias generadas por los responsables de la administración, y sería la primera probabilidad de ser fuente causal del éxito o fracaso de la organización, lo que descartaría a la globalización como la causa directa. 
El segundo nivel de planteamiento requiere de un entendimiento y un comprender objetivo, sobre la consideración a que si la globalización es un proceso, tal como viene siendo tratada o explicitada en certámenes o eventos por expertos, profesionales, políticos y lo sorprendente, incluso por especialistas de la administración en la dinámica de las organizaciones.

De esta manera trataremos someramente de dilucidar si la globalización es producto de lo imaginado, diseñado, planificado, programado y dirigido para que afecte a las personas, las organizaciones, a la sociedad y en su conjunto al mundo.

Tratándose de términos y conceptos, primero como una obligación referenciaremos al Diccionario de la Lengua Española [3] que sobre el proceso determina: «(Del latín processus) 3. Conjunto de las fases sucesivas de un fenómeno natural o de una operación artificial».

Para el desarrollo de este nivel de planteamiento tomaremos como referente básico al libro Introducción a la teoría general de administración [4] que cita lo planteado por Fayol, que el planear, organizar, dirigir, coordinar y controlar son los «elementos de la administración que constituyen el llamado proceso administrativo».

Asimismo, se anota que Henri Fayol (1841-1925) «vivió las consecuencias de la Revolución Industrial» y que tuvo una trayectoria exitosa en la administración, apreciando que la sumatoria de estos dos aspectos le sirvió como factor de impulso y/o motivador para formular su teoría, lo que le valió posteriormente el ser reconocido como el padre de la Administración.

Lo anecdótico en lo relacionado con nuestra temática central, es que en la actualidad se está repitiendo este episodio, de que están viviendo las personas y organizaciones las consecuencias de la Globalización.

Lo explicitado en los dos niveles de planteamiento, sea por su dinámica y resultados de las funciones administrativas o procesos de gestión pueda ser considerada a la Administración en causa o efecto de la Globalización, o por la denotación o connotación de proceso que se quiere dar, quedarían descartados.

El tercer nivel de planteamiento se da por post facto, por el cual se postularía a que la Globalización es una situación que aparece por efecto o consecuencia del desarrollo de la ciencia y la tecnología, en la que mayor opción de beneficio y de disfrute lo tienen los países desarrollados, los países ricos.

Lo que marcó la Revolución Industrial en el siglo XIX puede servir como un referente experiencial de la evolución histórica, social, científica y tecnológica que pasó la humanidad, por la cual no se le podría aducir que el cambio que originó en la dinámica de las organizaciones tuvo intencionalidad de generar el desempleo, la explotación cuantitativamente, selectiva y económica de los trabajadores, de incrementar la jornada laboral mediante la incentivación económica con propósitos de reducir su tiempo de descanso y disfrute que es lo que se espera del avance de la tecnología.

Este panorama en el siglo XXI se repite y se agrava con la globalización.

Asumiéndose que la Globalización es una situación causal por efecto o consecuencia del desarrollo de la ciencia y la tecnología, se convierte en impulsor o motivador para que las organizaciones ejecuten funciones administrativas y desarrollen una gestión efectiva para afrontar las consecuencias que afectan la dinámica individual y organizacional. 
A estos efectos se observa que la comunidad empresarial, industrial e institucional viene respondiendo exitosamente con propuestas, enfoques proactivos, técnicas y modelos de gestión, que en la mayoría de sus contenidos está presente la Psicología Organizacional.

El mayor logro de estos resultados lo obtienen los países desarrollados, sea porque poseen grandes recursos naturales, por su poder económico, tener el monopolio industrial o comercial.

Es necesario plantear una diferenciación de los países desarrollados, haciendo distinciones entre ellos, están los que se orientan a incrementar desmesuramente sus ganancias para lograr mayor dominio sobre los países no desarrollados, lo cual en el mejor de los casos regulan su derecho al desarrollo, en otros someterlos a ser solamente operadores de tecnología, quitándoles toda posibilidad a su aspiración de hacer investigación, y en el caso extremo, de convertir a los países a ser pobres y condenarlos a ser su mercado cautivo, de ser sólo consumidores de sus productos directamente o a través de terceros mediante la triangulación de empresas transnacionales.

Los efectos de la globalización, como consecuencia del desarrollo de la ciencia, contradictoriamente, nos alejan de la «realidad»; entendida ella como el espacio vital en el cual los seres humanos deberían convivir mejor con una amplia y libre socialización, sin discriminaciones ni exclusiones. Penosamente vemos más distante esta «realidad» en la medida que avanza aceleradamente la ciencia y la tecnología.

\section{MARCO REFERENCIAL}

Consideramos pertinente de bosquejar conjuntamente los alcances de la Administración y la acción coadyuvante que ejerce Psicología en el desarrollo de las organizaciones, que en un criterio interdisciplinario perspectivas que a manera de marco teórico sirvan para explicar y afrontar las consecuencias de la globalización.

En un sentido formal, una Teoría es un grupo ordenado de supuestos que se formulan con el objeto de explicar entre dos o más hechos observables, y las teorías nos ofrecen un enfoque estable para entender lo que experimentamos, estos enfoques o escuelas teóricas del pensamiento de la administración, se desarrollaron en secuencia histórica, que no sustituyen las ideas posteriores a las anteriores; es así que cada nueva escuela ha tenido que complementar a las anteriores o coexistir con ellas, por otra parte, cada escuela ha seguido evolucionando y algunas incluso, se han fusionado con otras.

En este accionar interdisciplinario entre la Administración y la Psicología, es necesario partir que corresponde a las organizaciones el afrontar los efectos de la globalización y enfrentar a un mercado exigente que cambia constantemente, y que para atender estas situaciones requiere que los responsables de la gestión sean más eficientes y eficaces.

Estos líderes organizacionales deberán aplicar plenamente sus capacidades y experiencia personal (aprendizajes), ante el escenario cambiante y altamente competitivo mediante una amplia visión, análisis ético, con sensibilidad frente a las necesidades sociales, al respeto de la diversidad cultural, el tener una idea clara y práctica efectiva del trabajo, de ejecutar interrelacionadamente los procesos de la planeación, organización, integración de personal, dirección y control orientados hacia la implementación de organizaciones inteligentes. 
Esta realidad de constante cambio fue lo que caracterizó la culminación del siglo xx, generando una nueva realidad de intensa exigencia para el inicio del siglo xxI que toma la posta en una época de inestabilidad económica, comercial, política y social para la administración.

Desde finales del siglo xIx se definía a la Administración como el proceso de planificar, organizar, coordinar, dirigir y controlar las actividades de los miembros de la organización y el empleo de todos los demás recursos organizacionales, con el propósito de alcanzar las metas establecidas por y para la organización.

A finales del siglo xx, el proceso administrativo bajo una perspectiva global considera cinco funciones básicas: la planificación, organización, «integración de personal», dirección y control de las actividades de los entes de la organización y el empleo de todos los recursos con el objeto de alcanzar las metas establecidas.

En la práctica, el proceso de administrar no entraña cuatro o cinco series de actividades independientes, ligeramente relacionadas, se deberá entender y aplicar como un grupo de funciones interrelacionadas (sistémico) que mantienen muy activos a los gerentes y que determinan la dinámica (gestión) de la organización como un todo (sinergia).

Ante este panorama, la administración en una perspectiva dinámica deberá tener como finalidad el dar forma y sentido de manera consciente y constante a las organizaciones, de contar con personal idóneo para alcanzar sus metas, con personas responsables que practiquen en su accionar una cultura del deber y del cumplimiento de sus funciones en su puesto de trabajo o nivel de gestión.

El éxito de una organización dependerá, en gran medida, de la calidad de sus supervisores, jefes, gerentes, ejecutivos, directivos. Ellos son los responsables de dirigir las actividades e influir en los trabajadores para lograr los objetivos, el cumplimiento de las obligaciones sociales, a ser que las organizaciones sean competentes, competitivas y establezcan su permanente desarrollo.

El referente básico aborda a la Administración en una perspectiva global mediante las funciones administrativas en un enfoque sistémico [5] (ver cuadro 1).

La Administración bajo un enfoque sistémico considera a la Planificación como una de las actividades principales de la administración para alcanzar las metas y el logro de los objetivos de una organización. Otra manera de definir a la planificación, es como el proceso para establecer metas y un curso de acción adecuado para alcanzarlas y, finalmente, la clasifica como estratégica, táctica y la operacional.

Una organización es un patrón de relaciones -muchas relaciones simultáneas, entrelazadas-, por medio de las cuales las personas, bajo el mando de los gerentes, persiguen metas comunes. Estas relaciones son producto de los procesos para tomar decisiones con el nombre de planificación.

Por una parte, los gerentes quieren estar seguros para que sus organizaciones duren mucho tiempo, y por otro lado los miembros de la organización necesitan un marco estable y comprensible (reglas de juego) en el cual puedan trabajar unidos para alcanzar las metas de la organización, es decir estables. 
Cuadro 1. Enfoque de Sistemas de la Administración.

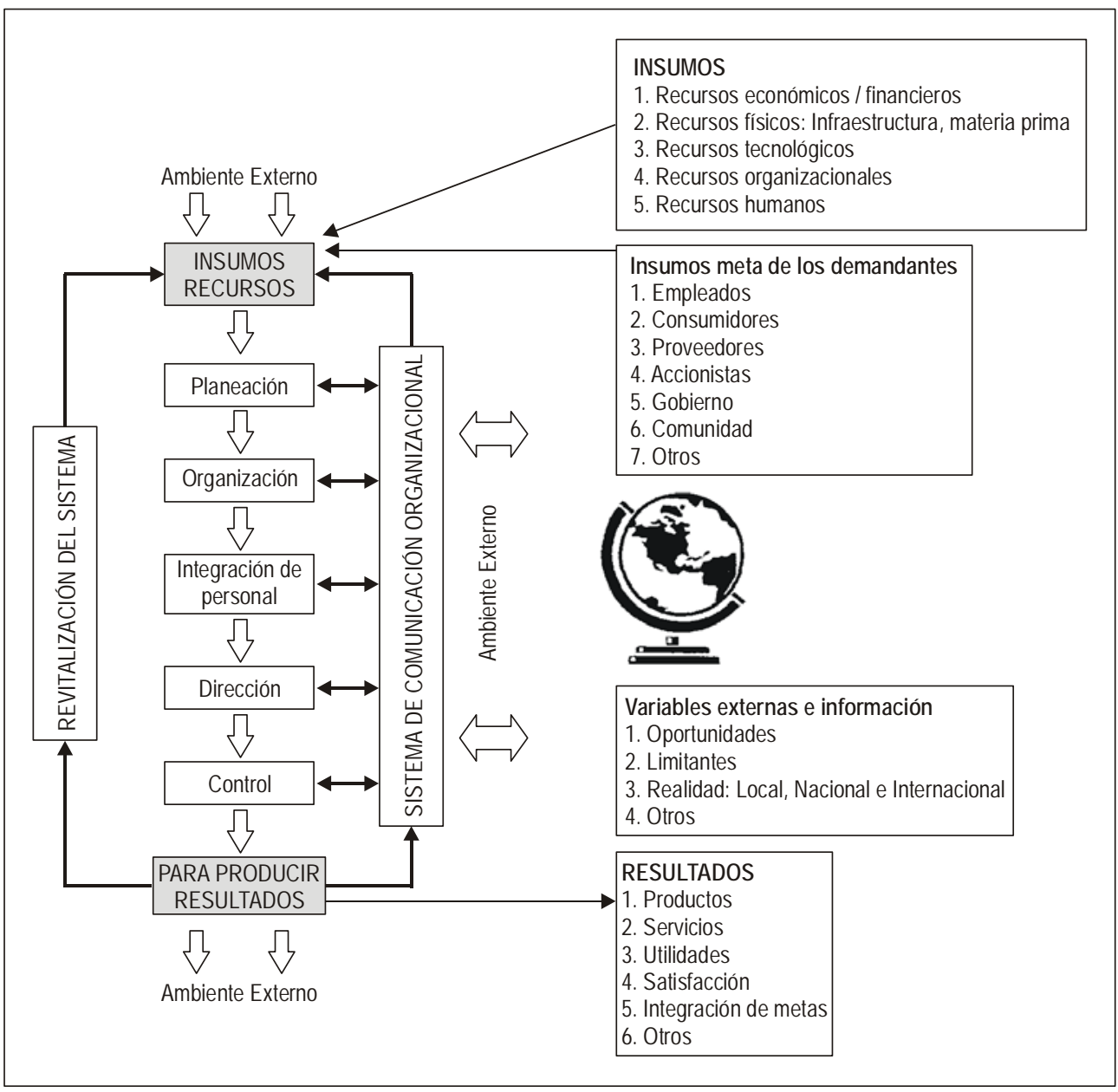

El proceso de organización determina la manera en que los gerentes dan forma a las relaciones, convirtiéndolas en estructuras organizacionales, y que conduzcan a los empleados hacia el futuro de la organización.

El proceso de organización es imprescindible para conocer las formas de realizar el diseño y la estructura más conveniente para la organización; igualmente la estrategia referida al personal, a la tecnología y a determinar las tareas de las organizaciones, la que ayudará a dividir, sistematizar y coordinar las actividades de la organización, de esta manera hacer que el diseño de la organización sea efectivo a la luz de las metas de la organización.

En el proceso de organización, el análisis de las reglas básicas permite a las personas trabajar dentro de la estructura de la organización, normas que se refieren a la autoridad y el gobierno; así como, para conservar una estructura de organización resistente y lo bastante vigorosa para que genere resultados: los productos de bienes y/o servicios nuevos. 
El proceso de Integración de personal o gestión de los recursos humanos se encarga de reclutar, seleccionar, preparar, evaluar y potenciar a las personas para que trabajen con eficiencia y eficacia dentro de las estructuras organizacionales.

Koontz y Weihrich determinan que el objetivo de la Integración de Personal es «garantizar que las funciones organizacionales sean desempeñadas por personal calificado idóneo y dispuesto a ejercerlas», y responde al principio de «cuanto más clara sea la definición de las funciones organizacionales y sus requerimientos humanos, y cuanto mejores sean las técnicas que se empleen en la selección, evaluación y capacitación de los administradores, tanto mayor será la calidad administrativa de una empresa».

Asimismo, esta función administrativa la definen como el «cubrir y mantener cubiertos los puestos de la estructura organizacional.», para lo cual se «...requiere la identificación de las necesidades de recursos humanos y de la ocupación en la estructura organizacional, y su conservación en este estado, con personas competentes.», y se logra «cuando desarrollamos una serie de acciones para identificar los requerimientos de la fuerza de trabajo, realizar un inventario del personal disponible y reclutar, seleccionar, contratar, remunerar, evaluar, planear las carreras, capacitar o desarrollar, y ascender en alguna otra forma tanto a los candidatos como a los titulares de los puestos, para que puedan cumplir con sus tareas de un modo eficaz y eficiente».

Otro término que se usa con frecuencia para la función administrativa de integración de personal es la»administración de los recursos humanos».

En esta perspectiva, encontramos a Bohlander, Snell y Sherman [6] enfatizando que «Las organizaciones en el mundo competitivo actual están descubriendo que lo que hace toda la diferencia es cómo se combinan los temas particulares de los Recursos Humanos. En general, los gerentes no se centran de manera aislada en aspectos de los Recursos Humanos como contratación, capacitación y compensaciones. Cada una de las tareas de recursos humanos se combina en un sistema global para mejorar la participación y la productividad de los trabajadores».

En esta perspectiva, hemos planteado un modelo de gestión de recursos humanos [7] que comprende procesos, cuya secuencialidad para su ejecución no es rígida, puede variar según la política de la organización, y programas, que por la temporalidad de su ejecución pueden ser cíclicos-eventuales como continuos-permanentes. Esta propuesta se puede apreciar gráficamente en el cuadro 2.

El proceso de Dirección es el esfuerzo administrativo para que el colectivo de los trabajadores se concentren en las metas de la organización; este proceso tiene el desafío que implica el imaginar un futuro deseable para la organización, y el poder llevarla hacia ese futuro, es una tarea central de la administración.

El proceso de Dirección asume el reto de establecer una dinámica eficiente, efectiva y eficaz en la ejecución de los procesos de la planificación y la organización, en el compromiso de lograr una exitosa gestión para la organización y sus miembros. 
Cuadro 2. Gestión sistémica de los recursos humanos: Componente psicológico.

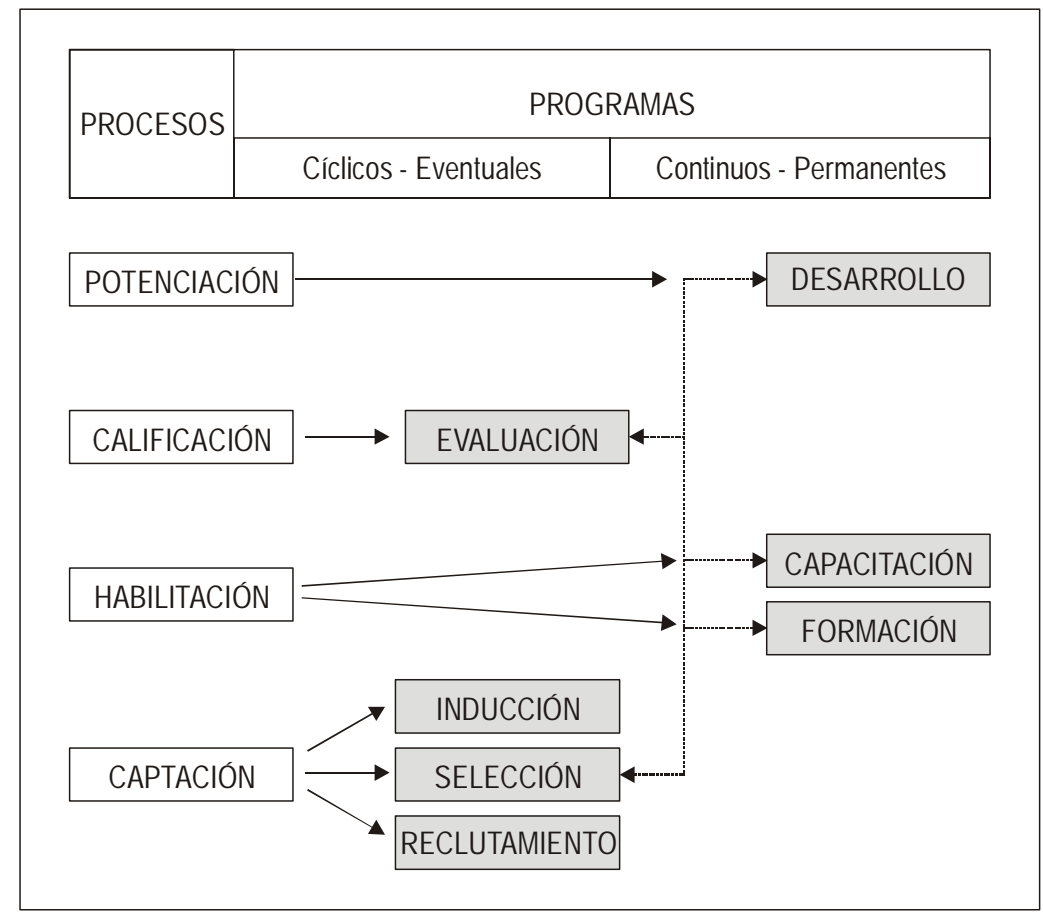

Elaboración propia

El proceso de control se encarga de velar para que se cumplan los procedimientos, asegurando que se está avanzando satisfactoriamente hacia las metas y que se están usando los recursos de la organización de manera eficiente.

Este proceso ayuda a los gerentes a monitorear la eficacia de la planificación, organización y dirección, así como la eficiencia de los recursos humanos, y para que se tomen las medidas correctivas necesarias.

\section{¿Qué tipo de Control debemos ejecutar? [8]}

Dependiendo de la política y/o recursos de la organización se puede optar por:

- Control Óptimo, que no es lograr un mayor porcentaje de control, sino un mejor control posible al menor costo (tiempo, dinero y esfuerzo).

Controlar el $100 \%$ normalmente no es lo deseable, por ser poco práctico y muy caro.

- Control Efectivo, está basado en la existencia de estándares que fijan los límites superior e inferior, entre los cuales considera como normal el rendimiento.

Controlar el $100 \%$ incluido lo que está «normal» no es lo deseable, por ser costoso (tiempo, dinero y esfuerzo). Por tanto, se recomienda controlar sólo lo anormal, es decir, todo aquello que sale de estos límites. 
Sin embargo, todo lo expuesto anteriormente no desemboca en la conclusión de que un plan estratégico razonable y una estructura organizativa sensata puedan alcanzar las metas de la organización, se requiere, además, asumir el reto gerencial orientado a la administración del cambio y la innovación organizacional. En este sentido, consideramos pertinente bosquejar los alcances de la Psicología en la disciplina de la Administración

Los gerentes y los investigadores de la administración llevan mucho tiempo suponiendo que las metas de la organización son inalcanzables, por ello es necesario que exista el compromiso permanente de sus miembros, lo que podrá lograrse mediante la motivación, una de las características de la psicología humana que contribuye al grado de compromiso de una persona, que considera los factores que ocasionan, canalizan y sustentan la conducta humana en un sentido particular y comprometido.

De igual forma el liderazgo es esencial para que los miembros del grupo trabajen juntos y que la ejecución de los planes estratégicos la efectúan personas que trabajan en un mundo de relaciones complejas y que pueden cambiar con el tiempo.

También sabemos que los grupos no son unidades que se cohesionan de manera automática, o que el flujo de información vital en ocasiones puede ser entorpecido, estos temas son atendidos por equipos (trabajo en equipo), así como por la comunicación y la negociación.

Asimismo, en la actualidad aparece con más fuerza el tema de «obligaciones sociales», concepto que compromete el cumplimiento no sólo de las metas y objetivos de las organizaciones, sino fundamentalmente de la comunidad en general. Hoy la teoría administrativa está surgiendo al amparo del compromiso social, la «responsabilidad social» de las organizaciones.

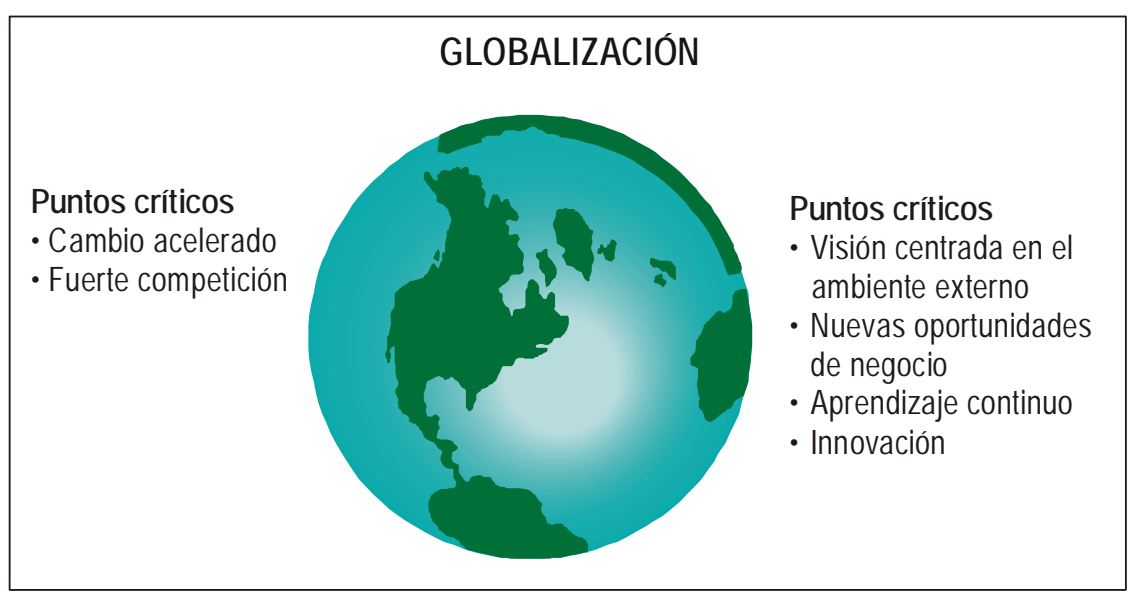


Efectos de la globalización en la administración de las organizaciones

\begin{tabular}{|c|c|c|}
\hline \multirow{2}{*}{$\mathrm{N}^{0}$} & \multicolumn{2}{|c|}{ CAMBIOS } \\
\hline & ANTES & TENDENCIA ACTUAL \\
\hline \multirow{2}{*}{01} & $\begin{array}{l}\text { Estructura Permanente } \\
\text { Organizaciones definitivas, } \\
\text { estáticas, rígidas y permanentes }\end{array}$ & $\begin{array}{l}\text { Estructura Flexible } \\
\text { Organizaciones provisorias, } \\
\text { efimeras, sueltas y dinámicas }\end{array}$ \\
\hline & $\begin{array}{l}\text { Mantenimiento del Status Quo. } \\
\text { Tradición. Visión para el Pasado }\end{array}$ & $\begin{array}{l}\text { Creatividad e Innovación. } \\
\text { Visión para el Futuro. }\end{array}$ \\
\hline \multirow[t]{2}{*}{02} & $\begin{array}{c}\text { Acción de Comando } \\
\text { basado en la autoridad jerárquica }\end{array}$ & $\begin{array}{c}\text { Acción Orientadora } \\
\text { basada en el liderazgo democratico }\end{array}$ \\
\hline & Ordenes e Instrucciones & Misión y Visión \\
\hline \multirow{3}{*}{03} & $\begin{array}{l}\text { Obediencia } \\
\text { Conformidad y obediencia } \\
\text { a las órdenes superiores }\end{array}$ & $\begin{array}{l}\text { Colaboración } \\
\text { Cooperación y colaboración } \\
\text { basados en el compromiso }\end{array}$ \\
\hline & $\begin{array}{l}\text { El jefe piensa, decide } \\
\text { e impone sus decisiones }\end{array}$ & $\begin{array}{l}\text { El lider, piensa, orienta, aconseja y asesora } \\
\text { a sus colaboradores }\end{array}$ \\
\hline & $\begin{array}{l}\text { Subordinados } \\
\text { Hacer, hacer siempre la misma cosa, } \\
\text { no pensar y no discutir }\end{array}$ & $\begin{array}{l}\text { Personas piensan, hacen, discuten, sugieren, } \\
\text { cooperan, colaboran, intentan, errar, crear, } \\
\text { innovar para lograr los objetivos }\end{array}$ \\
\hline \multirow{2}{*}{04} & $\begin{array}{l}\text { Actividad Muscular } \\
\text { Trabajo operativo, manual, servil, } \\
\text { rutinero, repetitivo y monótono }\end{array}$ & $\begin{array}{l}\text { Actividad Cerebral } \\
\text { Trabajo mental, cerebral, } \\
\text { creativo, innovador y desafiante }\end{array}$ \\
\hline & $\begin{array}{l}\text { Personas como } \\
\text { proveedores de Mano de Obra } \\
\text { Énfasis en el Músculo }\end{array}$ & $\begin{array}{l}\text { Personas como } \\
\text { proveedores de conocimiento. } \\
\text { Enfasis en el cerebro }\end{array}$ \\
\hline \multirow{3}{*}{05} & $\begin{array}{c}\text { Seguidores de Reglas } \\
\text { Empleados contratados y } \\
\text { que centran el punto diariamente }\end{array}$ & $\begin{array}{l}\text { Ser Emprendedores } \\
\text { Emprendedores internos o } \\
\text { externos que rompen reglas }\end{array}$ \\
\hline & $\begin{array}{l}\text { Reglas y regulamientos internos. Horario rígido de trabajo. } \\
\text { Jerarquía de autoridad. Descripción del cargo. } \\
\text { Evaluación del desempeño. Salario mensual fijo. }\end{array}$ & $\begin{array}{l}\text { Misiones, metas, Resultados a alcanzar. Énfasis en la } \\
\text { contribución. Medios quedan por cuenta de cada persona. } \\
\text { Participación en los resultados. Remuneración variable. }\end{array}$ \\
\hline & $\begin{array}{c}\text { Rutina } \\
\text { Burócratas conservadores } \\
\text { y tradicionalistas }\end{array}$ & $\begin{array}{c}\text { Ruptura } \\
\text { Emprendedores, creativos } \\
y \text { innovadores }\end{array}$ \\
\hline \multirow[t]{2}{*}{06} & $\begin{array}{c}\text { Especialización } \\
\text { Trabajo parcial, fragmentado, } \\
\text { dividido y especializado }\end{array}$ & $\begin{array}{l}\text { Multifuncionalidad } \\
\text { Trabajo variado, completo, } \\
\text { multifuncional y en equipo }\end{array}$ \\
\hline & $\begin{array}{c}\text { División del trabajo } \\
\text { y especialización }\end{array}$ & $\begin{array}{l}\text { Visión global del trabajo } \\
\text { y multifuncionalidad }\end{array}$ \\
\hline \multirow{3}{*}{07} & $\begin{array}{l}\text { Trabajo a tiempo completo } \\
\text { Trabajo realizado dentro de la } \\
\text { empresa en tiempo integral }\end{array}$ & $\begin{array}{l}\text { Trabajo de tiempo parcial } \\
\text { Trabajo realizado en cualquier } \\
\text { lugar y en tiempo parcial }\end{array}$ \\
\hline & $\begin{array}{l}\text { Concepto tradicional del empleo. Trabajo vitalicio y exclusivo } \\
\text { hasta jubilación. Contrato de trabajo. Horario definitivo y rígido } \\
\text { diario. Derecho de huelga. Descanso semanal y vacaciones anuales }\end{array}$ & $\begin{array}{l}\text { Nuevo concepto de trabajo: just in time. No precisa ser hecho diario } \\
\text { o periódicamente. Realizado en cualquier tiempo o lugar. Trabajo } \\
\text { remoto (home office) o cooperativas (workstations). Internet o Intranet. }\end{array}$ \\
\hline & $\begin{array}{c}\text { Personas como empleados } \\
\text { de una sola empresa }\end{array}$ & $\begin{array}{l}\text { Personas como proveedores de } \\
\text { actividades para muchos clientes }\end{array}$ \\
\hline \multirow[t]{2}{*}{08} & $\begin{array}{c}\text { Labor solitaria } \\
\text { Trabajo individual, aislado, } \\
\text { solitario y confinado socialmente }\end{array}$ & $\begin{array}{c}\text { Labor solidaria } \\
\text { Trabajo grupal, conjunto, } \\
\text { solidario en equipos integrados }\end{array}$ \\
\hline & Énfasis en la eficiencia individual & Énfasis en la sinergia grupal \\
\hline
\end{tabular}

Continúa.. 


\begin{tabular}{|c|c|c|}
\hline \multirow[t]{2}{*}{09} & $\begin{array}{c}\text { Las personas son administradas a través de la planeación. } \\
\text { Organización, dirección y control de recursos humanos. } \\
\text { Recursos son estáticos. Las personas son dependientes } \\
\text { de la empresa. }\end{array}$ & $\begin{array}{l}\text { Las personas ayudan a administrar los demás recursos: } \\
\text { financieros, materiales, informáticos, tecnológicos, etc. } \\
\text { Las personas son colaboradores del negocio: toman decisiones, } \\
\text { diagnostican situaciones, sirven al cliente.. }\end{array}$ \\
\hline & Personas como recursos empresariales & Personas como socios del negocio \\
\hline \multirow{3}{*}{10} & $\begin{array}{c}\text { Gerentes } \\
\text { Jefes autocráticos y controladores }\end{array}$ & $\begin{array}{c}\text { Líderes } \\
\text { Líderes democráticos y motivadores }\end{array}$ \\
\hline & $\begin{array}{l}\text { Conocimiento técnico. Especialización en una función. } \\
\text { Posición jerárquica. Cargo definido. Lograr ahorros }\end{array}$ & $\begin{array}{c}\text { Conocimiento humanista. Visión sistémica } \\
\text { Visión del cliente. Trabajo en equipos. Crear nuevos negocios }\end{array}$ \\
\hline & $\begin{array}{l}\text { Mantener los negocios de la empresa } \\
\text { para obtener lucros y ahorros }\end{array}$ & $\begin{array}{l}\text { Generar nuevos negocios para } \\
\text { mantener la empresa en el mercado }\end{array}$ \\
\hline \multirow{3}{*}{11} & $\begin{array}{c}\text { Capital financiero } \\
\text { Balance contable, numérico y tradicional }\end{array}$ & $\begin{array}{c}\text { Capital Intelectual } \\
\text { Balance patrimonial del conocimiento }\end{array}$ \\
\hline & $\begin{array}{l}\text { Balance del patrimonio físico. Énfasis en dados numéricos. } \\
\text { Evaluación del pasado. Activos tangibles y materiales. } \\
\text { Énfasis en recursos financieros y materiales }\end{array}$ & $\begin{array}{c}\text { Balance del patrimonio global. Énfasis en el valor agregado. } \\
\text { Prospección del futuro. Activos intangibles e invisibles. } \\
\text { Énfasis en el conocimiento. }\end{array}$ \\
\hline & $\begin{array}{c}\text { Resultados financieros de la empresa } \\
\text { Hacer }\end{array}$ & $\begin{array}{c}\text { Resultados intangibles de la empresa } \\
\text { Pensar }\end{array}$ \\
\hline
\end{tabular}

Elaboración propia.

\section{MÉTODO}

La diversidad conceptual, la complejidad en lo denotativo o connotativo y sus consecuencias en los modelos por su diversa intencionalidad que se contraponen para efectos de evaluar, han determinado a la presente investigación como exploratoria.

Esta realidad plantea la necesidad de evaluar y sistematizar propuestas para incrementar las investigaciones en base a propuestas sobre la efectividad de las intervenciones para el afrontamiento a los efectos de la globalización.

\section{Evaluación y Propuesta}

Se considera que la Globalización es una situación causal por efecto o consecuencia del desarrollo de la ciencia y la tecnología, y se convierte en motivadora del cambio en las organizaciones para que ejecuten funciones administrativas y desarrollen una gestión efectiva para afrontar las consecuencias que afectan la dinámica individual y organizacional, intentaremos graficar los efectos principales de la globalización en la administración.

En el campo de la planeación, tomaremos lo tratado sobre «El planeamiento estratégico en la perspectiva psicológica como producto de los procesos cognoscitivos para la dinámica humana y organizacional» [9] que plantea:

«La temática del Planeamiento Estratégico está generalmente identificada por su práctica en el campo de la administración, como una de las herramientas valiosas de gestión en la dinámica de las organizaciones. Esta situación resulta más que paradójica en el trabajo de los profesionales de la salud, particularmente en el campo de la Psicología, al no darle la misma importancia que le otorgan los otros campos profesionales de administración e ingeniería, restringiendo el alcance de su aplicabilidad en el plano existencial de la dinámica humana».

Esta realidad implica que debe brindarse un mayor conocimiento sobre la herramienta de gestión de uso obligado en las grandes organizaciones de los países desarrollados y generalizado en las organizaciones de los países en vías de desarrollo, para propiciar su uso 
en el campo individual como un medio para lograr mejor los objetivos de su plan de vida, para una eficiente y eficaz dinámica humana (misión) y la satisfacción de estar alcanzando el deseado estado ideal de éxito personal (visión).

Tratar esta temática en una perspectiva psicológica tiene como propósito facilitar la reflexión y recordar que el Planeamiento Estratégico para su elaboración y efectiva aplicación, necesita como recursos-insumos a los trascendentales procesos cognoscitivos del pensamiento, percepción, memoria e imaginación.

Entre estos procesos cognoscitivos, el pensamiento tiene prevalencia en razón a que:

- $\quad$ Es un proceso que está socialmente condicionado, lo que posibilita a tener conciencia de lo que acontece en la realidad que lo rodea y con los contextos de su interés que desea relacionarse.

- $\quad$ Su naturaleza estratégica de referenciar el pasado y visualizar el futuro, estando en el presente.

- Tiene capacidad de procesar información y conocimiento en cantidad y calidad.

- Crea mecanismos de autoevaluación en un marco normativo axiológico comportamental.

Esto determina la aplicabilidad del Planeamiento Estratégico como una herramienta útil en el plano individual, que siendo el Psicólogo responsable del manejo científico de los procesos psicológicos, específicamente del proceso cognoscitivo del pensamiento es la autoridad profesional para las intervenciones en personas y organizaciones de producción de bienes y servicios En la disciplina psicológica su aplicación también es de gran utilidad al campo clínico, educativo, social comunal y político.

Es necesario enfatizar que la persona siempre forma parte de una organización sea en condición activa como pasiva; desde su nacimiento se integra a la organización familiahogar, que continúa durante su crecimiento y desarrollo transitando en otras organizaciones informales: nivel barrial, y formales: la escuela, la universidad, centro laboral.

En condición pasiva, antes del nacimiento es parte de la organización biológica de la madre y cuando muere forma parte de la organización de un cementerio, que según su estatus económico recibirá diferentes niveles de calidad de atención, y si en el mundo fue creyente irá a formar parte de organizaciones bien definidas por su misión, visión y objetivos estratégicos, como son el cielo, infierno u otras organizaciones en tránsito.

Otra aplicación como intervención del Psicólogo es el uso de una técnica preparatoria o de inducción para brindar ayuda a los miembros de las organizaciones, sea cual fuere su nivel de desempeño laboral. Esta técnica es de gran utilidad para despertar interés y sensibilización individual o en conjunto para que participen en las funciones del Planeamiento, y me estoy refiriendo a la herramienta identificada como Método de Casos, que sirve como una forma de entrenar las personas para pensar estratégicamente.

Al respecto, las organizaciones (empresas- industrias-instituciones), en su perspectiva para enfrentar el siglo xXI, dentro de un contexto globalizado y de ser parte de la dinámica de la sociedad del conocimiento, vienen realizando esfuerzos orientados a convertirse en organizaciones inteligentes [10], estar en esta situación les permitirá a unas ser exitosas, y otras a supervivir en el mercado tan competitivo, exigente y cambiante. 
El referenciar al Método de Casos [11] tiene como propósito dar a conocer una de las técnicas que posibilitan entrenar al cerebro para pensar inteligentemente, de esta manera dar el primer paso si es que el empleado o ejecutivo aspira a ser miembro inteligente de una organización inteligente.

La aplicación de la técnica del Método de Casos posibilita asumir una actitud permanente hacia el aprendizaje, a emerger la iniciativa y la creatividad en las personas, sean estudiantes o profesionales, trabajadores, supervisores, jefes, gerentes, ejecutivos o directivos, es decir, utilizar el método de casos como una herramienta para la toma de decisiones, cuya práctica permanente se convertirá en una forma de comportamiento orientada a convertirse en gestores de organizaciones inteligentes.

El Psicólogo por su formación integral -científica y humanista- ejerce autoridad sobre el manejo del proceso cognoscitivo del pensamiento, insumo/recurso indispensable para elaborar y aplicar el Planeamiento Estratégico; asimismo, por su praxis profesional coadyuva tanto al desarrollo de las personas, de las organizaciones haciéndolas más efectivas, más competitivas, y al desarrollo nacional en su labor de elevar los niveles de eficiencia, eficacia y mejora de la calidad de vida de los trabajadores (Población Económicamente Activa)». [12]

En el campo de los recursos humanos, tomaremos lo tratado en Perea J. L [7] que plantea el proceso de calificación de recursos humanos a través de sus tres programas:

«El Proceso de Calificación se instrumentaliza tradicionalmente mediante el Programa de Evaluación de personal, que tiene como objetivo de conocer las iniciativas que el trabajador ha desarrollado y que ha significado mejora cuantitativa y cualitativamente en los procesos de trabajo o de productos, es decir, aportes distintos al rendimiento en el puesto de trabajo.

Los acelerados cambios que impone la globalización, la competitividad y las exigencias de los usuarios del mercado interno y externo, las organizaciones están aperturando variantes en el tradicionalmente conocido Programa de Evaluación de personal, que hasta el momento se aplican básicamente en tres tipos de pruebas de evaluación exploratoria referidas a:

Evaluación del desempeño del trabajador

Evaluación de los méritos del trabajador

Evaluación de la actuación del trabajador.
(Rendimiento)

(Innovación, creatividad)

(actitudes)

La evaluación de méritos tiene varios propósitos:

«Motivacional», que propicia una cultura organizacional de creatividad entre los trabajadores;

«Incentivadora», que promueva una cultura integrativa y participativa de esfuerzo compartido para el beneficio común entre los trabajadores;

«Exploratoria», que posibilita comportamientos orientados a la implementación o al mantenimiento de una cultura organizacional axiológica.

Según la política de la organización, el aspecto compensatorio por los aportes (creatividad, innovaciones, mejoramiento de procesos o procedimientos, etc.) puede ser material y / o económico. 
La evaluación de méritos refuerza la creatividad y la evaluación de desempeño o del rendimiento refuerza la productividad.

La evaluación de la actuación tiene como objetivo conocer el nivel actitudinal de los trabajadores hacia la organización.

El diseño de evaluación de la actuación toma en cuenta objetivos específicos de la política de la organización relacionados a su crecimiento o desarrollo.

La finalidad es conocer y seleccionar a colaboradores de confianza que lleven a cabo la ejecución de la política de expansión y competitividad de la organización. Es la búsqueda interna de aliados estratégicos.

La evaluación de la actuación tiene las características siguientes:

- Evaluativa exploratoria sobre los niveles de identificación e internalización del trabajador con la cultura y política de la organización;

- Selectiva para detectar y proponer personal como aliados estratégicos de la organización para garantizar las acciones o campañas de crecimiento y desarrollo de la organización.

El proceso de potenciación de recursos humanos está orientado a descubrir, estimular y poner en práctica las cualidades y potencialidades del personal que tiene la responsabilidad de conducir grupos humanos debidamente organizados (supervisores, jefes, gerentes, ejecutivos, directivos). Se diseñan y ejecutan Programas de Desarrollo de personal, cuyos eventos deberán brindar nuevas técnicas, enfoques, herramientas e instrumentos que faciliten la obtención de logros exitosos en la gestión.

Hay organizaciones que consideran en su política de desarrollo empresarial / institucional el programa de Formación de Cuadros, que permite asistir a a los trabajadores que no ejerciendo una responsabilidad de gestión, y que tienen antecedentes sobresalientes tanto en sus evaluaciones de rendimiento-desempeño, de actuación, de méritos, en eventos de formación y capacitación, y calificativos de ingreso a la organización.

\section{CONCLUSIONES}

Es necesario resaltar los esfuerzos que actualmente los líderes políticos vienen desarrollando a través de propuestas para posibilitar el acercamiento entre los países desarrollados y pobres con el propósito de afrontar las consecuencias de la globalización en la aldea mundial, que trata de lograr ayuda de índole económico, político, comercial, sanitario, educativo, social, ecológico, etc., para los países más vulnerables.

En estas situaciones propositivas juegan gran papel las organizaciones macro (países) para formular e implementar sus políticas de Estado o gubernamentales como las organizaciones de producción de bienes y servicios (empresas, industrias, instituciones) para proceder a los cambios necesarios en su administración y gestión.

Se vienen produciendo cambios significativos, desde el cambio de paradigmas en el campo de las negociaciones en general, desde los esquemas ganador-perdedor al de ganador-ganador, relaciones laborales-convenios, relaciones comerciales (contratos de concesión, tratados), relaciones políticas, sociales, etc. 
A nivel internacional, tenemos los grandes trabajos que hizo como Jefe de Estado el Papa Juan Pablo II, al poner en práctica el $5^{\circ}$ Dogma Mariano («Lady of all Nations», aprobado mediante Concilio del 02 de julio de 1951), plasmados en los esfuerzos de mediador, conciliador, intercedor y defensor de los países más pobres.

A nivel nacional, en el año 2004, se plantearon diversas propuestas de cambio, una de ellas es la de Álvaro Vargas LLosa, que podríamos considerarla como alternativa de un outsider, y que aparece en su libro Rumbo a la Libertad: por qué la Izquierda y el Neoliberalismo fracasan en América Latina [13].

Su propuesta como líder de autoexilio, denominación que fuera manejada por los analistas políticos y medios de comunicación, propicia una sociedad libre con la práctica de los principios y valores humanos.

La otra propuesta que podríamos considerar como alternativa del insider es la que aparece en el libro de Alan García Modernidad y política en el siglo ххı: Globalización con justicia Social, que propicia la concertación [14].

Cabe precisar, que Alan García, actualmente como Presidente Constitucional de la República, viene planteando cambios, cuyo propósito está hacia la implementación de programas sociales de no exclusión de los más pobres, hacia el cambio responsable en las políticas gubernamentales, de la práctica de la cultura del deber, del cumplimiento, y de fomento del desarrollo de las zonas más deprimidas hacia una producción exportadora.

Los efectos de la globalización, como consecuencia del desarrollo de la ciencia, contradictoriamente nos alejan de la «realidad»; entendida ella como el espacio vital en el cual los seres humanos deben convivir mejor con una amplia y libre socialización, sin discriminaciones ni exclusiones, y que penosamente, vemos más distante esta «realidad» en la medida que la tecnología avanza aceledaramente.

Las organizaciones vienen a ser el contexto social laboral de los seres humanos y el soporte productivo de la sociedad, y en ellas, no obstante la presencia de la tecnología, no le garantiza su existencia; solamente el factor humano es el que decide su supervivencia, su desaparición o su éxito.

El pragmatismo pretende «ayudar» al plantearse como la alternativa para poder afrontar retos y desafíos, hacia los cambios acelerados, la competitividad, comunicación satelital, países sin fronteras, crecimiento económico sin desarrollo social, etc.,) y sin notarlo se ingresa a la vorágine de la tecnocracia sin rostro humano.

De todo lo expuesto, se invoca a establecer un compromiso de trabajar sea profesionalmente, técnica o manualmente, hacia una perspectiva de ejecución de Políticas de Estado que trascienda a las políticas gubernamentales, y manera de colofón traer a la memoria de todos la expresión virtual de Ciro Alegría que «el mundo es ancho y ajeno».

\section{REFERENCIAS BIBLIOGRÁFICAS}

1. Senge M., P. (1999). La danza del cambio: Una herramienta para la quinta disciplina. Ed. Norma.

2. Perea R., J. L. (2005). «Sistematización en una reforma curricular». Revista de Investigación en Psicología, Vol. 8, No 2. Lima: Instituto de Investigaciones Psicológicas, Facultad de Psicología, UNMSM. 
3. Real Academia Española. Diccionario de la lengua española. $23^{\mathrm{a}}$ ed.

4. Chiavenato, I. (1999). Introducción a la teoría general de la administración. Ed. McGraw-Hill.

5. Koontz, H. / Weihrich, H. Administración: Una perspectiva global. $10^{\mathrm{a}}$ ed. Edit. McGraw-Hill.

6. Bohlander, Snell y Skerman (2001). Administración de Recursos Humanos. 12 a ed. International Thomson Editores.

7. Perea R. J. L. (2006). «Gestión de recursos humanos; Enfoque sistémico en una perspectiva global». Revista de Investigación en Psicología, Vol. 9, N 1 . Lima: Instituto de Investigaciones Psicológicas, Facultad de Psicología, UNMSM.

8. Perea R. J. L. (2004). Separata curso «Principios y técnicas de administración». Lima: Facultad de Psicología, UNMSM.

9. Perea R, J. L. (2006). «El planeamiento estratégico: En la perspectiva psicológica como producto de los procesos cognoscitivos para la dinámica humana y organizacional». Edición: Psicología tópicos de Actualidad de la Escuela Académico-Profesional de la Lima: Facultad de Psicología, UNMSM.

10. Senge M., P. La quinta disciplina: El arte y práctica de la organización abierta al aprendizaje. Ed. Granica.

Senge M., P. La quinta disciplina en la práctica: Estrategias y herramientas para construir la organización abierta al aprendizaje. Ed. Granica.

11. ESAN. El método de casos. (AN176).

12. Perea R. J. L. (2004). Separata curso «Psicología organizacional». Lima: Facultad de Psicología, UNMSM.

13. Vargas Llosa, Álvaro (2004). Rumbo a la libertad: por qué la izquierda y el neoliberalismo fracasan en América Latina. Editorial Planeta.

14. García Pérez, Alan (2004). Modernidad y política en el siglo ххı: Globalización con justicia social. Editorial Matices. 